\title{
A prospective 15-year follow up of 154 consecutive patients with trigeminal neuralgia treated by percutaneous stereotactic radiofrequency thermal rhizotomy.
}

\author{
Commentary
}

Chad J. Morgan, M.D. and John M. Tew JR., M.D.

Department of Neurosurgery, University of Cincinnati College of Medicine, Cincinnati, Ohio

Our 1995 publication $^{1}$ defined prospective outcomes that could be expected for patients with trigeminal neuralgia (TN) who underwent percutaneous stereotactic rhizotomy (PSR). A decade after its publication, this article continues to be a guiding principle for our practice. In an era when some neurosurgeons are moving away from percutaneous procedures for TN, its attributes cannot be overlooked. Our continued experience demonstrates that for those patients in whom dense hypalgesia is achieved, a substantive pain-free interval is provided with an acceptably low rate of dysesthesia.

We conclude that PSR is the preferred treatment of patients with TN who are not candidates for posterior fossa procedures because of risks or personal preference. Our continued experience demonstrates that more than $95 \%$ of patients are satisfied with their outcomes after PSR; however, approximately $25 \%$ of patients have required retreatment during this 15-year follow-up period. Of more than 3000 patients treated, an excessive sensory deficit remains the only significant cause of dissatisfaction associated with PSR, and there have been no deaths or serious side effects.
The concluding comments found in the article are simple: "rates of pain recurrence and dysesthesia correlate with the severity of the lesion." To master the operative procedure is to master the art of obtaining dense hypalgesia. Although surgeons are sometimes inclined to focus on the technical and anatomical aspects of procedures, we encourage them to take the time to hone the skills required to create the art. In doing so, they will find PSR to be among the most rewarding aspects of their practice. Throughout my (J.M.T.) neurosurgical career, this procedure has remained one of the most satisfying forms of therapy that is offered to patients. With minor modifications in technique and equipment, PSR continues to be a therapy of major importance for the effective control of one of life's most horrifyingly painful conditions.

\section{References}

1. Taha JM, Tew JM Jr, Buncher CR: A prospective 15-year follow up of 154 consecutive patients with trigeminal neuralgia treated by percutaneous stereotactic radiofrequency thermal rhizotomy. J Neurosurg 83: 989::993, 1995 
Authors 
Title

Neurosurg. Focus / Volume 18 / April, 2005 
Authors 